\title{
Giant Nevus Lipomatosus Cutaneous Superficialis on the Buttock
}

\author{
Masayasu Takegawa, MD \\ Natsuko Kakudo, MD, PhD \\ Naoki Morimoto, MD, PhD \\ Masakatsu Hihara, MD \\ Hiromu Masuoka, MD \\ Kenji Kusumoto, MD, PhD
}

\begin{abstract}
Summary: Nevus lipomatosus cutaneous superficialis is a benign hamartoma characterized by the presence of mature ectopic adipocytes in the dermis. Here, we report a case of giant nevus lipomatosus cutaneous superficialis, measuring $24 \times 14 \mathrm{~cm}$ in diameter, on the buttock. The tumor was resected, and the cutaneous defect was closed with a rotation flap under general anesthesia. No complication or recurrence occurred after surgery. Giant nevus lipomatosus cutaneous superficialis is very rare, and repair of the skin defect required plastic and reconstructive surgery. (Plast Reconstr Surg Glob Open 2018;6:e1918; doi: 10.1097/GOX.0000000000001918; Published online 21 November 2018.)
\end{abstract}

$\mathrm{N}$ evus lipomatosus cutaneous superficialis (NLCS) is a benign hamartoma characterized by the presence of mature ectopic adipocytes in the dermis. ${ }^{1,2}$ NLCS was first described in 1921 by Hoffman and Zurhelle ${ }^{3}$ and has been classified into 2 clinical forms: classical and solitary. ${ }^{1}$ The classical type is usually composed of multiple groups of skin-colored, pedunculated nodules. The solitary type is characterized by a solitary dome-shaped or sessile papule or nodule. ${ }^{1,4}$

A giant classical NLCS lesion measuring $24 \times 14 \mathrm{~cm}$ in diameter on the buttock was resected, and the cutaneous defect was closed with a rotation flap. Giant NLCS of this size is very rare, and no case has previously been reported in the plastic surgery field.

\section{CASE}

The patient was a 48-year-old male who presented with a huge tumor on his left buttock. The size was small when he first noticed it 30 years ago, but it gradually grew to $24 \times 14 \mathrm{~cm}$ in diameter. During this period, the patient never consulted any medical institute as he had no symptoms. On admission, the tumor was mulberry in shape on the surface, blackish-salmon pink in color, and elastic soft (Fig. 1; see figure, Supplemental Digital Content 1, which displays the tumor observed from the posterior side, http://links.lww.com/PRSGO/A851). Magnetic resonance imaging (MRI) revealed that the tumor contained unevenly distributed fat and had infiltrated

From the Department of Plastic and Reconstructive Surgery, Kansai Medical University, Osaka, Japan.

Received for publication June 22, 2018; accepted July 11, 2018.

Copyright @ 2018 The Authors. Published by Wolters Kluwer Health, Inc. on behalf of The American Society of Plastic Surgeons. This is an open-access article distributed under the terms of the Creative Commons Attribution-Non Commercial-No Derivatives License 4.0 (CCBY-NC-ND), where it is permissible to download and share the work provided it is properly cited. The work cannot be changed in any way or used commercially without permission from the journal. DOI: 10.1097/GOX.0000000000001918 the subcutaneous tissue [Fig. 2; see figure, Supplemental Digital Content 2, which displays a horizontal view on MRI T2 (yellow arrow indicates the tumor), http:// links.lww.com/PRSGO/A852). Incisional skin biopsy was performed 2 times, and the histopathological analysis demonstrated soft fibroma and seborrheic keratosis. The tumor with the pedicle of $11 \times 6 \mathrm{~cm}$ in size was resected with a $1-\mathrm{cm}$ resection margin over the fascia (see figure, Supplemental Digital Content 3, which displays a tumor after it was resected with a $1-\mathrm{cm}$ resection margin on the fascia, http://links.lww.com/PRSGO/A853). The skin and cutaneous defect of $12 \times 7 \mathrm{~cm}$ were closed with a rotation flap (Fig. 3; see figure, Supplemental Digital Content 4, which displays elevation of the rotation flap on the buttock, http://links.lww.com/PRSGO/A854). Ectopic mature adipocytes were found in the tumor dermis on histopathological examination (Fig. 4). Based on the clinical and pathological findings, it was diagnosed as NLCS. No recurrence of the nodule was noted as of 6 months postoperatively.

\section{DISCUSSION}

NLCS is considered to be a rare nevus and has been reported in a few case reports. However, its etiology remains unclear.

The histopathology of NLCS usually includes proliferation of ectopic mature adipocytes in the reticular dermis, comprising $10-50 \%$ of the lesion. ${ }^{4}$ Theories, such as mesenchymal perivascular differentiation of lipoblasts, focal heterotopic development of adipose tissue, and adipose metaplasia throughout degenerative changes in

Disclosure: The authors have no financial interest to declare in relation to the content of this article. The Article Processing Charge was paid for by the authors.

Supplemental digital content is available for this article. Clickable URL citations appear in the text. 


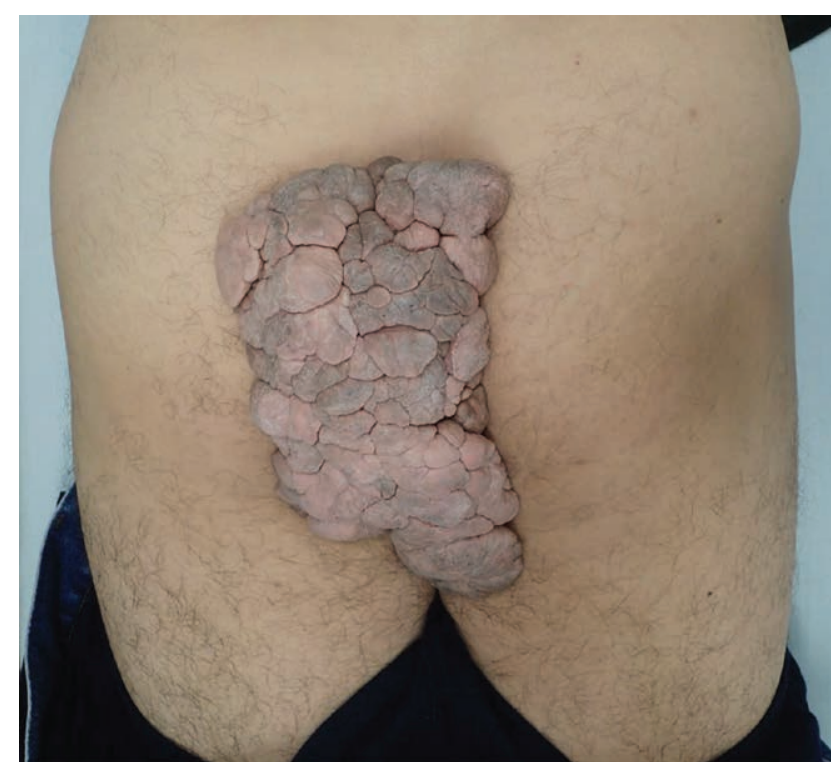

Fig. 1. Frontal view of the tumor.

dermal collagen bundles and elastic tissue, have been proposed for the heterotopic occurrence of adipose tissue, but they have yet to be substantiated. ${ }^{5}$

Pathological tissue from NLCS is likely to be confused with plexiform neurofibroma, connective tissue nevus, vascular malformation, lipomatosis, or lipoblastomatosis. In the present patient, soft fibroma with seborrheic keratosis was suspected based on the preoperative partial biopsy of the tumor surface, but NLCS was definitively diagnosed by pathological examination after total resection.

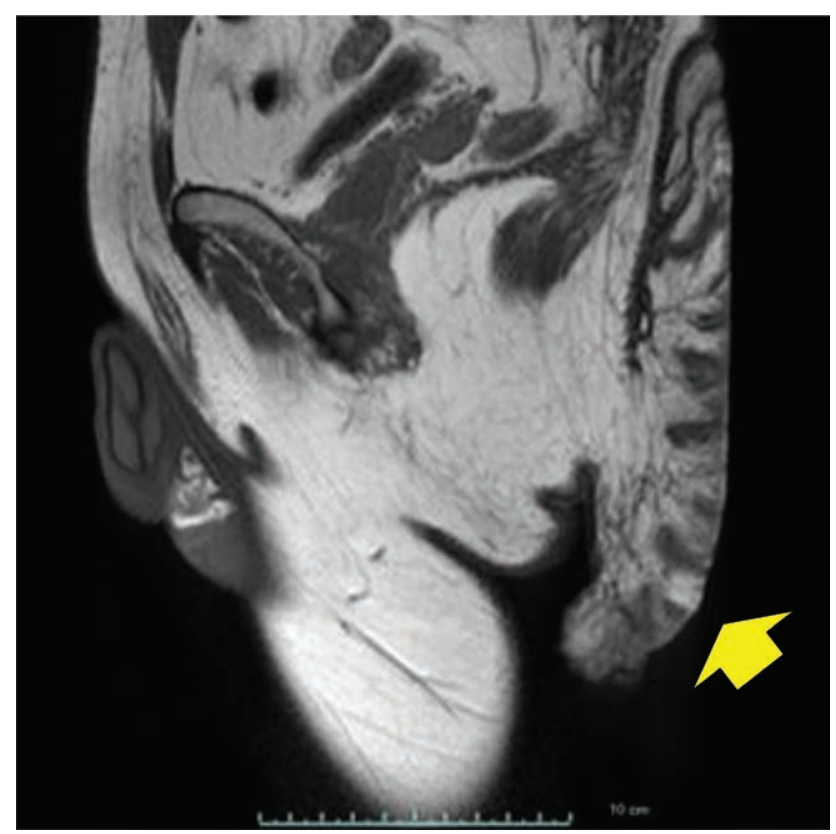

Fig. 2. Sagittal view on MRIT1 (yellow arrow indicates the tumor).

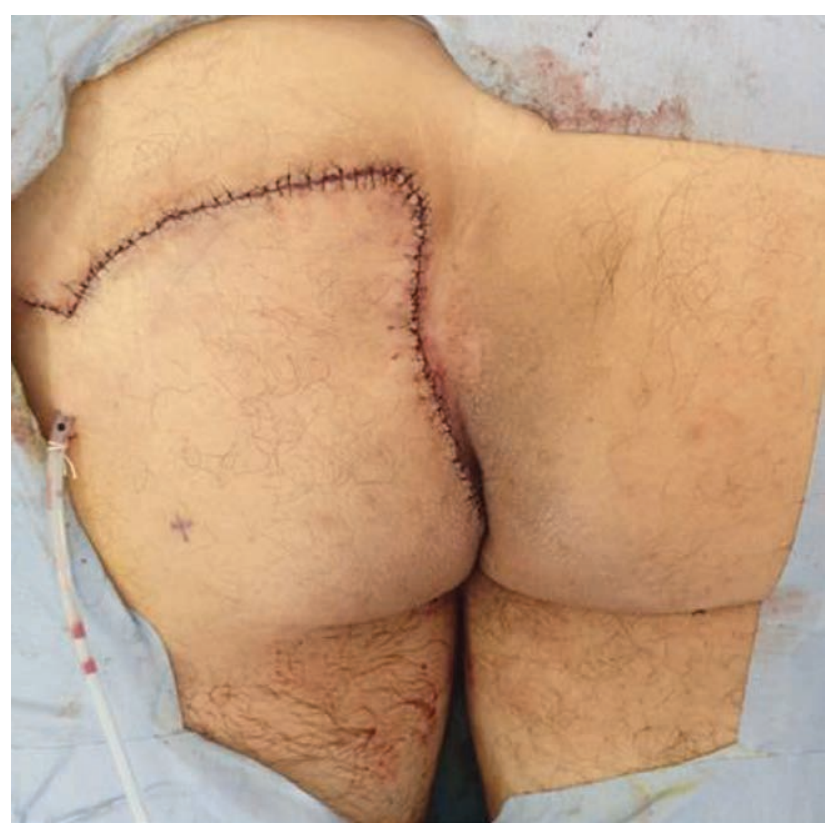

Fig. 3. HE staining.

NLCS has been classified into 2 clinical forms: classical and solitary. ${ }^{1,3}$ The classical type often grows to be large in size. ${ }^{5}$ A case of NLCS on the buttock that was $40 \times 28 \mathrm{~cm}$ in size was previously reported as the largest. ${ }^{6}$ Two other cases of NLCS of approximately $20 \times 30 \mathrm{~cm}$ in size have also been reported ${ }^{7,8}$ but no case has been reported from the plastic surgery field, and the resection defect of our patient was closed with a flap procedure, differing from other cases. The characteristics of giant NLCS are a tumor on the lumbar or buttock area and a long disease duration. ${ }^{5}$ It is often compressed while sitting and enclosed under clothing for long periods. In general, giant tumors on the occipital to back and buttock areas remain untreated for long periods because the patients cannot see it themselves and do not notice it. Surgical resection is the first choice for treatment of NLCS. Cryotherapy is applied only for patients who do not want surgery, but the outcome is only partially satisfactory. ${ }^{6}$ Although the giant NLCS itself exhibited no symptoms, sitting became difficult for the patient, and he decided to visit the hospital. For treatment of NLCSs, correct pathological diagnosis and complete resection with resection margins are needed, especially for giant NLCSs, which often require wide skin resection and closure of the skin defect with skin flaps.

\section{PATIENT CONSENT}

The patient provided written consent for the use of the images.

Natsuko Kakudo, $M D, P h D$

Department of Plastic and Reconstructive Surgery

Kansai Medical University

2-5-1, Shin-machi, Hirakata

Osaka 573-1010, Japan

E-mail: kakudon@hirakata.kmu.ac.jp 


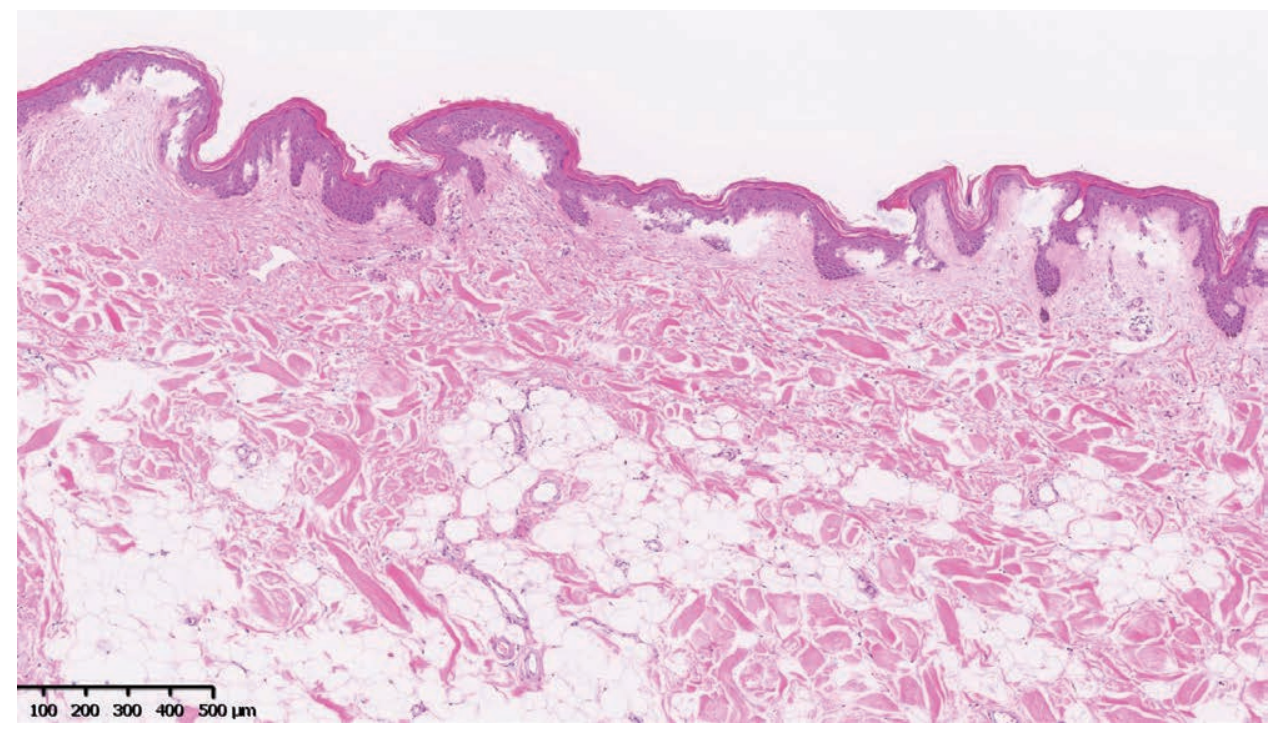

Fig. 4. Immediately after coverage of the skin defect with a rotation flap during surgery.

\section{REFERENCES}

1. Leung AKC, Barankin B. Nevus lipomatosus superficialis on the left proximal arm. Case Rep Dermatol Med. 2017;2017:6908750.

2. Das D, Das A, Bandyopadhyay D, et al. Huge nevus lipomatosus cutaneous superficialis on back: an unusual presentation. Indian J Dermatol. 2015;60:296-297.

3. Hoffman E, Zurhelle E. Ubereinen nevus lipomatodes cutaneous superficialis der linkenglutaalgegend. Arch dermatol Syph. 1921;130:327-333.

4. Lima CDS, Issa MCA, Souza MB, et al. Nevus lipomatosus cutaneous superficialis. An Bras Dermatol. 2017;92:711-713.
5. Kaneko T, Shiraishi M, Nomura K. A case of giant nevus lipomatosus cutaneous superficialis. J JOCD. 1999;53: 237-239.

6. Khandpur S, Nagpal SA, Chandra S, et al. Giant nevus lipomatosus cutaneous superficialis. Indian J Dermatol Venereol Leprol. 2009;75:407-408.

7. Al-Mutairi N, Joshi A, Nour-Eldin O. Naevus lipomatosus cutaneous superficialis of Hoffmann-Zurhelle with angiokeratoma of Fordyce. Acta Derm Venereol. 2006;86:92-93.

8. Knuttel R, Silver EA. A cerebriform mass on the right buttock. Dermatol Surg. 2003;29:780-781. 